\title{
Effect of Evidence-based Nursing on Perioperative Nursing of Patients with Gastric Cancer
}

\author{
QING QING. ZHU, HUI FANG. ZHANG, ZHEN QI.WEI, MIN. ZHAO, YING. LIU1, XIAO DONG. YANG AND XIAO HUI. \\ $\mathrm{CHENG}^{2 *}$ \\ Department of Gastrointestinal surgery, 1Department of Neursurgery, 2Nursing quality management division, The Second \\ Affiliated Hospital of Soochow University, No. 1055 Sanxiang Road, Suzhou, Jiangsu 215004, China
}

Zhu et al.: Effect of perioperative nursing on patients with gastric cancer

\begin{abstract}
To explore the therapeutic effect of evidence-based nursing on the perioperative care of gastric cancer patients. 81 patients with gastric cancer hospitalized in our department from September 2018 to September 2019 were randomly divided into 40 experimental group and 40 control group; the control group used conventional nursing intervention and the experimental group used evidence-based nursing intervention to compare the surgical index, mental and emotional, clinical index, visual analogue scoring system score and complications of the two groups. The results showed that there were no statistically significant differences in intraoperative bleeding and operation time between the two groups, and the amount of intraoperative fluid replenishment was significantly lower in the experimental group than in the control group; after the nursing intervention, the Self-rating Anxiety Scale and Self-rating Depression Scale scores of the experimental group were lower than those of the control group at $1 \mathrm{~d}$ preoperatively and 3 d postoperatively $(\mathbf{p}<\mathbf{0 . 0 5})$; the Self-rating Anxiety Scale and Self-rating Depression Scale scores of the experimental group were lower than those of the control group at $6 \mathrm{~h}, 24 \mathrm{~h}$ and $72 \mathrm{~h}$ postoperatively $(p<0.05)$. The visual analogue scoring system scores were all lower than those of the control group $(p<0.05)$; the time of getting out of bed after surgery, the time of first postoperative exhaustion, the time of postoperative ureter extraction, the time of early feeding, the time of semi-liquid diet, and the time of postoperative hospitalization were all shorter in the experimental group than in the control group, and the costs were lower than those of the control group, and the differences were statistically significant $(p<0.05)$. The comparison between groups was significantly different $(p<0.05)$. The clinical application of evidencebased nursing care in the perioperative nursing coordination of gastric cancer radical surgery can not only reduce postoperative complications, but also shorten hospitalization time and help promote patients recovery, which is worth promoting.
\end{abstract}

Key words: Evidence-based nursing, gastric cancer, perioperative period, VAS score

Gastric cancer, as a common malignant tumor, has the characteristics of high incidence rate, low survival rate and 5 y survival rate, which seriously endangering the health of patients. Most patients with gastric cancer need surgical treatment, especially radical gastrectomy. Although the curative effect is exact, there are many complications such as gastric bleeding, partial infection and delayed gastric emptying, which are not conducive to the recovery of patients prognosis ${ }^{[1]}$. In addition, cancer and surgical treatment can easily lead to panic and depression, which may interfere with the neuroendocrine function of patients and cause internal environment disorder, thus affecting the smooth progress of anesthesia and surgery, and also have adverse effects on the prognosis of patients ${ }^{[2]}$. Postoperative wound swelling and pain can cause insomnia, depression and even depression, and prolong the hospitalization time of patients ${ }^{[3]}$. Evidence based nursing $(\mathrm{EBN})$ refers to nursing staff working out a reasonable and relatively perfect nursing scheme based on scientific research evidence, clinical experience, patients' wishes and actual situation, and specific and specific problems encountered in clinical practice ${ }^{[4]}$. Therefore, in this study, we used evidence-based nursing to test its effect on perioperative anxiety and postoperative pain in patients with gastric cancer. 


\section{PATIENTS AND METHODS}

\section{Patient's collection}

A total of 81 gastric cancer patients from September 2018 to September 2019 in our hospital were selected and randomly divided into experimental group ( $\mathrm{n}=41$ cases) and control group ( $\mathrm{n}=40$ cases).

Inclusive criteria: The diagnostic criteria and site criteria of gastric cancer refer to the diagnostic and therapeutic criteria of gastric cancer ${ }^{[5]}$; Age $<75$ y old; Gastric cancer diagnosed by gastroscopy and pathological biopsy before operation and received laparoscopic Radical surgery (D2) operation; No obvious invasion and distant metastasis of tumor confirmed by enhanced Computed tomography (CT) of upper abdomen; No history of preoperative chemotherapy; No history of upper abdominal surgery; No history of diabetes; All patients underwent elective D2 surgery.

Exclusion criteria: Laparoscopic conversion, tumor dissemination and metastasis, palliative surgery, emergency surgery, combined organ resection, incomplete data. The experiment was examined and approved by the hospital ethics committee. All patients agreed and signed the informed consent.

In the control group, there were 8 cases of gastric fundus or cardia, 13 cases of gastric body, 19 cases of gastric antrum, age of $(56.5 \pm 6.8)$ y old, 18 cases of female and 22 cases of male; the experimental group: 6 cases of gastric fundus or cardia, 15 cases of gastric body, 20 cases of gastric antrum, age $(55.8 \pm 5.2)$ y old, including 20 cases of female and 21 cases of male. After statistical analysis, there were no significant differences in age, sex ratio, TNM stage, operation mode and location between the two groups ( $>>0.05$ ), as shown in Table 1.

\section{Methods}

The main steps and measures of the control group were as follows: after admission, the patients were given preoperative education and nutrition screening by the competent doctors and nurses, the patients were given clean enema the night before operation and on the $d$ of operation and the patients were instructed to fast $12 \mathrm{~h}$ before operation and $6 \mathrm{~h}$ before operation.

During the operation, patients were given general anesthesia and placed gastrointestinal decompression tube, and 2 abdominal drainage tubes were placed routinely, without limiting the amount of liquid input. Within $24 \mathrm{~h}$ after the operation, the patient-controlled intravenous analgesia pump was used, parenteral nutrition was given after operation, and liquid diet was given after anal exhaust. After 3-4 d of bed rest, patients were encouraged to get out of bed early. The specific activity time, intensity and frequency were reasonably arranged by patients according to their own conditions.

The patients in the experimental group were given evidence-based nursing plan, which were as follows:

The evidence-based nursing group was composed of the responsible nurse, the nurse in charge and the head nurse. The head nurse served as the team leader and was responsible for supervising and managing the work of the team, while the team members were responsible for consulting the relevant literature, determining the difficulties and priorities of nursing cooperation, and formulating nursing measures.

Cooperation of itinerant nurses: Based on the tumor size, location and surrounding organ invasion of patients, the relevant instruments, including sterile distilled water, medical bio glue and surgical film, were prepared, and the shadow less lamp lighting was adjusted in time to obtain a clear surgical field, keep the suction tube unobstructed, timely suck out the bleeding and exudate, and reduce the exfoliated cancer cells, To avoid contamination of abdominal cavity, and to observe the operation process closely, timely replace the contaminated dressings and instruments.

\section{TABLE 1: PATIENT'S BASELINE DATA}

\begin{tabular}{|c|c|c|c|c|}
\hline Factors & $\begin{array}{l}\text { Experience } \\
\text { group }(n=41)\end{array}$ & $\begin{array}{l}\text { Control } \\
\text { group } \\
(n=40)\end{array}$ & $x^{2}$ & $\mathrm{p}$ \\
\hline \multicolumn{5}{|l|}{ Gender } \\
\hline Male & 21 & 22 & \multirow[t]{2}{*}{0.904} & \multirow[t]{2}{*}{0.937} \\
\hline Femal & 20 & 18 & & \\
\hline Age (y) & $55.8 \pm 5.2$ & $56.5 \pm 6.8$ & 1.221 & 0.846 \\
\hline \multicolumn{5}{|l|}{$\begin{array}{l}\text { Location of } \\
\text { lesion }\end{array}$} \\
\hline Fundus or cardia & 6 & 8 & \multirow{3}{*}{2.256} & \multirow{3}{*}{0.668} \\
\hline Gastric body & 15 & 13 & & \\
\hline $\begin{array}{l}\text { Antrum of } \\
\text { stomach }\end{array}$ & 20 & 19 & & \\
\hline \multicolumn{5}{|l|}{ TNM staging } \\
\hline I & 8 & 10 & \multirow{4}{*}{3.521} & \multirow{4}{*}{0.216} \\
\hline II & 15 & 12 & & \\
\hline III & 11 & 12 & & \\
\hline IV & 7 & 6 & & \\
\hline \multicolumn{5}{|l|}{ Operation mode } \\
\hline $\begin{array}{l}\text { Proximal } \\
\text { gastrectomy }\end{array}$ & 8 & 9 & \multirow{3}{*}{3.037} & \multirow{3}{*}{0.317} \\
\hline $\begin{array}{l}\text { Distal } \\
\text { gastrectomy }\end{array}$ & 18 & 20 & & \\
\hline $\begin{array}{l}\text { Total } \\
\text { gastrectomy }\end{array}$ & 15 & 11 & & \\
\hline
\end{tabular}

Special Issue 1, 2021 
Equipment nurse cooperation: strictly distinguish the "tumor free area" and "tumor area", keep the gauze dry in the cancer area, use gauze pad to protect the surrounding tissues when the tumor is removed, and timely handle the gloves and gauze contaminated by the tumor. In addition, the instruments used before and after tumor resection should be placed separately, and the removed lymph nodes should not be directly contacted with hands. The lymph nodes should be arranged on the moist gauze. After the tumor was removed and the digestive tract was reconstructed, the surgical instruments were replaced. Before closing the abdomen, the abdominal cavity was rinsed with 1000-3000 $\mathrm{ml}$ distilled water for 2-4 times, and the residual liquid was sucked out with aspirator to prevent infection.

Prevention of complications: during the operation, oxygen saturation and vital signs of the patients were closely observed, the distribution of large vessels in the upper abdomen was understood, and the methods of vascular anastomosis or repair were mastered. First aid items should be prepared before operation, such as vascular suture, artery clip and rubber sling. In case of vascular injury during operation, timely cooperate with doctors for vascular anastomosis or repair.

\section{Observation index}

Operation indexes: operation time, intraoperative blood loss and fluid infusion volume. Self-rating Anxiety Scale (SAS) and self-rating Depression Scale (SDS) evaluation: SAS and SDS scores were performed in the two groups at 9:00 a.m. $3 \mathrm{~d}$ before operation, $1 \mathrm{~d}$ before operation and 3 da after operation. The score $\geq 50$ points indicated that the patients had anxiety or depression. The higher the score, the more serious the anxiety or depression. Visual analogue scale (VAS) score: VAS was used at $6 \mathrm{~h}, 24 \mathrm{~h}$ and $72 \mathrm{~h}$ after operation, and VAS pain score was given by the responsible nurse. 0 score was no pain and 10 score was severe pain. The use of postoperative analgesics and patients' satisfaction were counted $1 \mathrm{~d}$ before discharge. Clinical indicators: anal exhaust time, early eating time, postoperative ambulation time, eating semi liquid food time, hospitalization expenses, postoperative hospital stay, postoperative catheter removal time. Complications: the incidence of postoperative complications, including gastric bleeding, anastomotic leakage and pulmonary infection, were observed in the two groups

\section{Statistical analysis}

SPSS 20.0 software was used for statistical analysis in this study. The measurement data was expressed as $\mathrm{X} \pm \mathrm{SD}, \mathrm{t}$ test was used, count data was expressed as $\%$ and $\chi 2$ test was used. $\mathrm{p}<0.05$ was considered to be statistically significant

\section{RESULTS AND DISCUSSION}

There was no significant difference in intraoperative blood loss and operation time between the two groups. Intraoperative fluid infusion volume in the experimental group was significantly lower than that in the control group, as shown in fig 1.

There was a certain degree of anxiety and depression in the experimental group and the control group $3 \mathrm{~d}$ before the nursing intervention, but there was no statistical significance $(p>0.05)$. After the implementation of nursing intervention, the SAS and SDS scores of the experimental group at $1 \mathrm{~d}$ before operation and $3 \mathrm{~d}$ after operation were markedly lower than those of the control group $(\mathrm{p}<0.05)$, as shown in fig 2 .

VAS scores of the experimental group at $6 \mathrm{~h}, 24 \mathrm{~h}$ and $72 \mathrm{~h}$ after operation were obviously lower than those of the control group $(\mathrm{p}<0.05)$, as shown in fig 3 .
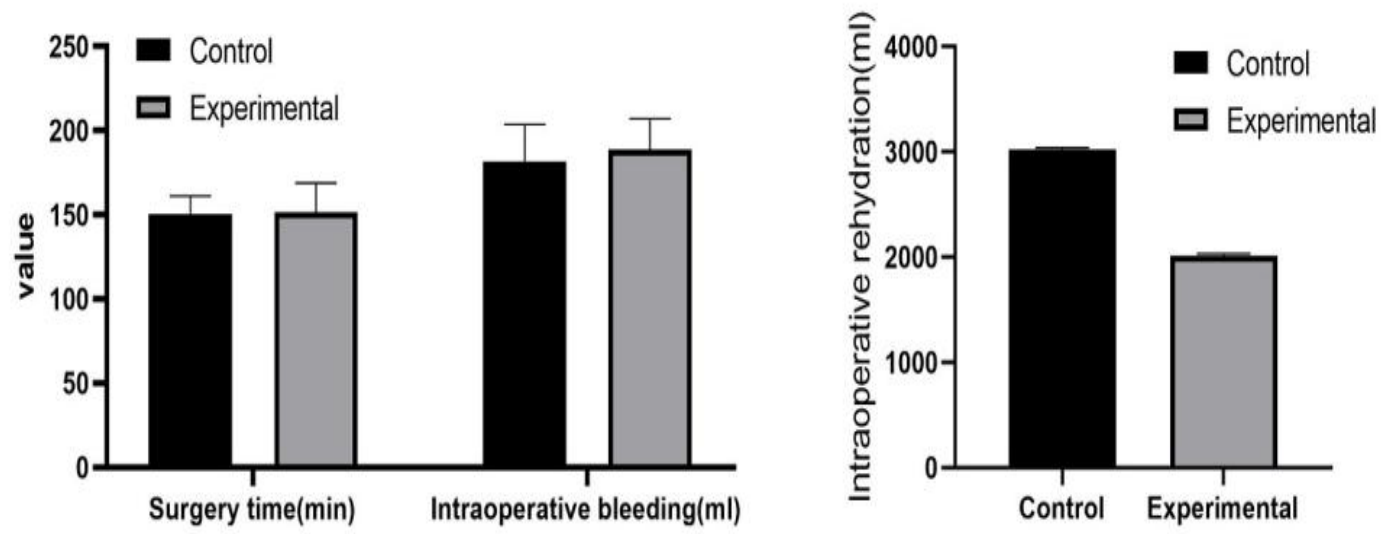

Fig. 1: Comparison of operation indexes between the two groups 

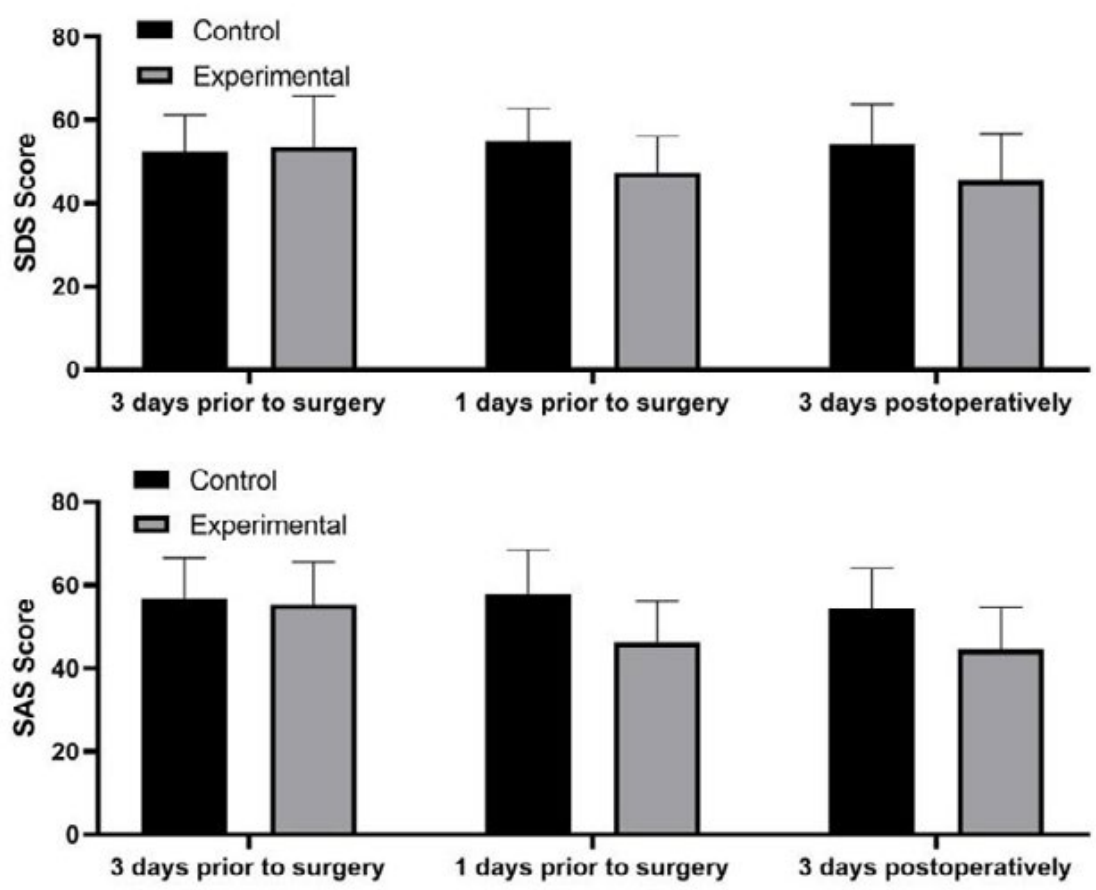

Fig. 2: Comparison of anxiety and depression between the two groups

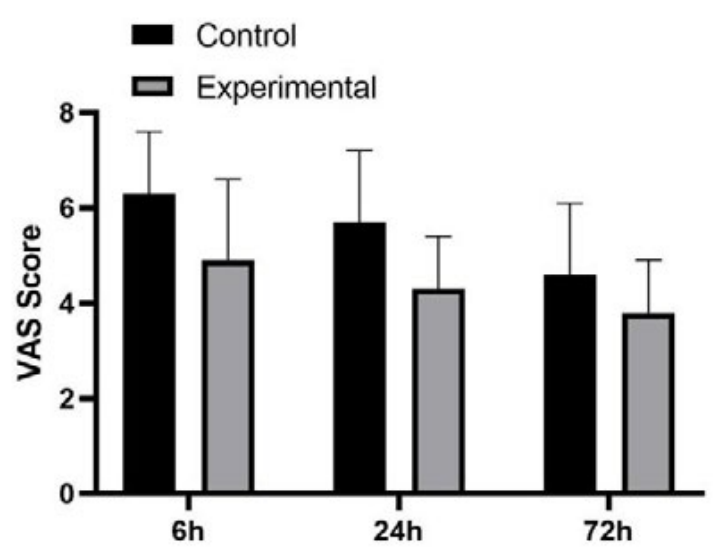

Fig. 3: Comparison of postoperative pain between the two groups

The time of getting out of bed after operation, the time of first exhaust after operation, the time of extubation after operation, the time of early eating, the time of entering semi liquid food and the length of hospital stay in the experimental group were significantly shorter than those in the control group, and the cost was significantly less than that in the control group $(\mathrm{p}<0.05)$, as shown in fig 4 .

Compared with the control group, the incidence of complications in the experimental group was significantly lower $(\mathrm{p}<0.05)$, as shown in fig 5 .

Gastric cancer, as a common malignant tumor of digestive tract, is prone to produce greater psychological pressure on patients, especially insomnia, depression, and even depression among people who lack knowledge of gastric cancer, thus affecting the therapeutic effect and even aggravating the disease ${ }^{[6]}$. According to the different degree of anxiety of patients before operation, consulting relevant literature and combining with clinical experience, psychological intervention was taken to explain gastric cancer related knowledge for patients, so that patients can understand the disease more clearly, reduce the psychological burden and worry of patients, help patients correct their wrong ideas, and face the disease more confidently ${ }^{[7]}$.

As a new nursing mode, evidence-based nursing can improve the pertinence, scientific and rationality of nursing work by combining clinical practice and various scientific basis ${ }^{[8-10]}$. Some studies have found that although radical gastrectomy can achieve good short-term efficacy, but the long-term recurrence rate is high (about $50 \%-70 \%$ ). Therefore, strengthening the cooperation of tumor free operation and nursing, strictly distinguishing the "tumor free area" and "tumor area", and placing the equipment according to classification and doing a good job of isolation can reduce the cancer cell shedding, so as to better prevent postoperative recurrence ${ }^{[11]}$. Meanwhile, distilled water can lyse tumor cells and reduce or inactivate tumor cells. Therefore, before closing the abdomen, wash the abdominal cavity in time to make the incision surface fully contact with the flushing fluid, and use the aspirator to absorb the residual liquid, which can avoid damaging the peritoneal cavity and reduce the growth 

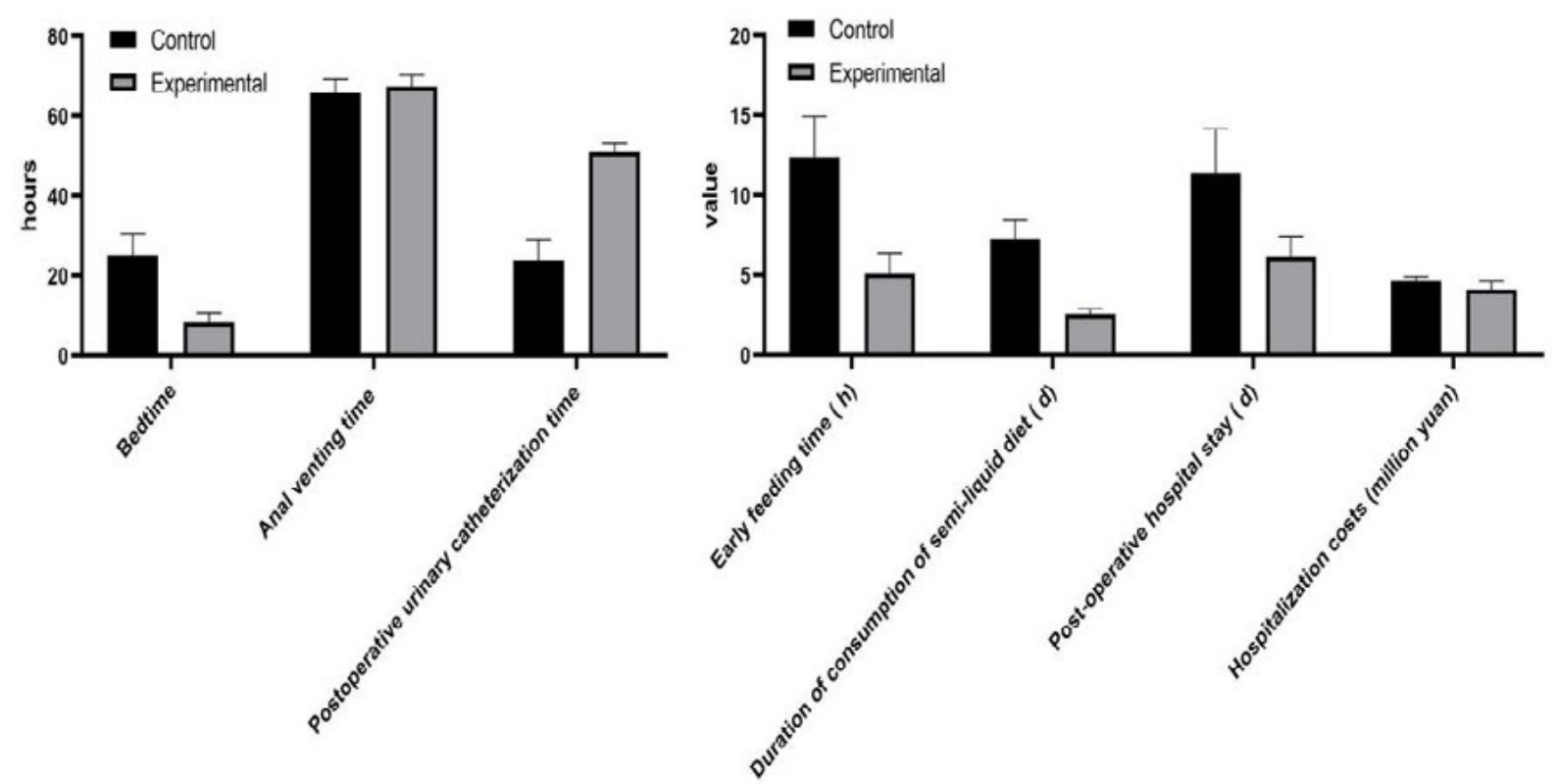

Fig. 4: Comparison of clinical indexes between the two groups

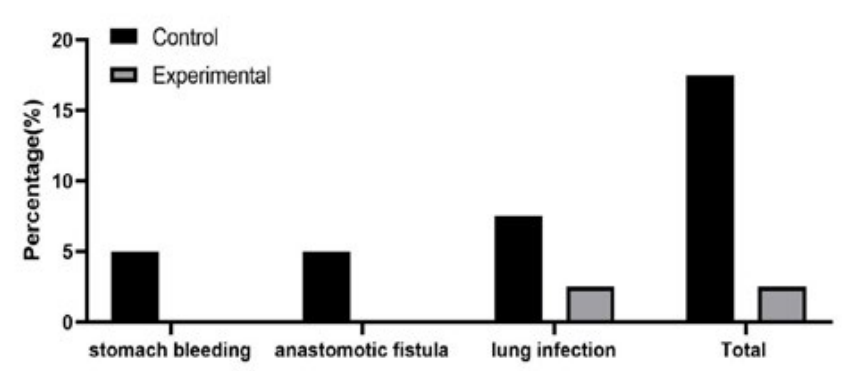

Fig. 5: Complications of the two groups

of cancer cells ${ }^{[12]}$. In addition, clearing the blood vessels and lymph nodes around the organs is a key factor in radical gastrectomy for gastric cancer. However, when lymph nodes are removed, lymph leakage is easy to occur. Therefore, once lymphatic vessel injury is found during operation, timely suture treatment can reduce the risk of lymphatic leakage to a certain extent ${ }^{[13]}$. In this study, the out of bed activity and hospitalization time of the experimental group were shorter, and the complications were less. This result was consistent with the previous research reports ${ }^{[14,15]}$, indicating that evidence-based nursing can reduce improper operation during operation and obtain better therapeutic effect.

Evidence based nursing is mainly based on more than ten optimization measures before, during and after the operation. Through preoperative communication between doctors and patients, good analgesia, improvement of fluid management, early eating and getting out of bed activities, evidence-based nursing can reduce or reduce the stress reaction of operation, so as to achieve the purpose of rapid recovery and early discharge of patients ${ }^{[16]}$. Intestinal tract is the largest immune organ in human body and one of the central organs of stress response. The intestinal peristalsis began to return to normal $6 \mathrm{~h}$ after abdominal surgery. Prolonged use of gastric tube increased the incidence of nausea, vomiting and pulmonary infection in patients with gastric cancer. Therefore, gastric tube was not routinely used in the experimental group. Oral administration of appropriate amount of carbohydrate before operation and early food intake after operation can avoid the stress reaction caused by starvation, promote the early recovery of intestinal function, maintain the function of intestinal mucosa, prevent flora imbalance and translocation, improve nutritional status and reduce the incidence of intra-abdominal and intra-abdominal complications ${ }^{[17]}$. Early postoperative recovery of diet can also reduce pneumonia, lower extremity deep vein thrombosis and other complications promote anabolism, does not increase the incidence of anastomotic leakage ${ }^{[18]}$.

Furthermore, the perioperative treatment of evidence-based nursing can significantly shorten the hospitalization time of patients, save hospitalization expenses, accelerate the turnover of beds in the Department, reduce the medical cost, so as to improve the utilization rate of health resources and reduce the economic burden of patients and their families.

Pain, as a subjective feeling of human body to noxious stimulation, contains physiological and emotional factors, which can cause sympathetic excitations such as increased heart rate, elevated blood 
pressure, gastrointestinal function inhibition, etc. Psychologically, the patients' recovery is affected by anxiety and uneasiness due to pain discomfort. Our results showed that EBC group (experimental group) could reduce anxiety and depression of patients before and after operation more than that of the conventional nursing group (control group). The VAS score of $6 \mathrm{~h}$, $12 \mathrm{~h}$ and $24 \mathrm{~h}$ after operation was lower than that of the general nursing group. Patient's satisfaction was also higher in the evidence-based nursing group, and the use of painkillers in the evidence-based nursing group was also less than that in the conventional nursing group.

To sum up, the clinical application of evidence-based nursing in the perioperative nursing cooperation of radical gastrectomy for gastric cancer can not only reduce postoperative complications, but also shorten the length of hospital stay, which is helpful to promote the rehabilitation of patients, and is worthy of promotion.

\section{Acknowledgements:}

This work was supported by The Second Affiliated Hospital of Soochow University, Qing Qing Zhu and Hui Fang Zhang contributed equally to this work.

\section{Conflict of interests:}

The authors declared no conflict of interest.

\section{REFERENCES}

1. Fearon KC, Ljungqvist O, Von Meyenfeldt M, Revhaug A, Dejong $\mathrm{CH}$, Lassen $\mathrm{K}$, et al. Enhanced recovery after surgery: a consensus review of clinical care for patients undergoing colonic resection. Clin Nutr 2005;24:466-77.

2. Lassen K, Coolsen MM, Slim K, Carli F, de AguilarNascimento JE, Schäfer M, et al. Guideline for perioperative care for pancreaticoduodenectomy: enhanced recovery after surgery (ERAS $®)$ society recommendations. Clin Nutr 2012;31:817-30.

3. Coolsen MM, Wong-Lun-Hing EM, van Dam RM, van der Wilt AA, Slim K, Lassen K, et al. A systematic review of outcomes in patients undergoing liver surgery in an enhanced recovery after surgery pathways. HPB 2013;15:245-51.

4. Mortensen K, Nilsson M, Slim K, Schäfer M, Mariette C, Braga $\mathrm{M}$, et al. Consensus guideline for enhanced recovery after gastrectomy: enhanced recovery after surgery (ERAS $®$ ) society recommendations. Br J Surg 2014;101:1209-29.

5. Yang Z, Zheng Q, Wang Z. Meta-analysis of the need for nasogastric or nasojejunal decompression after gastrectomy for gastric cancer. Br J Surg 2018;95:809-16.
6. Park DJ, Lee HJ, Kim HH, Yang HK, Lee KU, Choe K, et al. Predictors of operative morbidity and mortality in gastric cancer surgery. Br J Surg 2005;92(9):1099-102.

7. Kodera Y, Sasako M, Yamamoto S, Sano T, Nashimoto A, Kurita A. Identification of risk factors for the development of complications following extended and superextended lymphadenectomies for gastric cancer. Br J Surg 2005;92: 1103-9.

8. Mangram AJ, Horan TC, Pearson ML, Silver LC, Jarvis WR. Guideline for Prevention of Surgical Site Infection, 1999. Centers for Disease Control and Prevention (CDC) Hospital Infection Control Practices Advisory Committee. Am J Infect Control 1999;27:97-32.

9. Doglietto GB, Papa V, Tortorelli AP, Bossola M, Covino M, Pacelli F. Nasojejunal tube placement after total gastrectomy: a multicenter prospective randomized trial. Arch Surg 2004;139:1309-13.

10. Seo HS, Song KY, Jeon HM, Park CH. The impact of an increased application of critical pathway for gastrectomy on the length of stay and cost. J Gastric Cancer 2012;12:126-31.

11. Kreys ED, Koeller JM. Documenting the benefits and cost saving of a large multistate cancer pathway program from a payer's perspective. J Oncol Pract. 2013;9:e241-7.

12. Jones DE, Sutcliffe K, Pairman J, Wilton K, Newton JL. An integrated care pathway improves quality of life in primary biliary cirrhosis. QJM 2008;101:535-43.

13. Association Japanese Gastric Cancer. Gastric cancer treatment guidelines 2014. Gastric Can 2017;20:1-19.

14. Heslin MJ, Latkany L, Leung D, Brooks AD, Hochwald $\mathrm{SN}$, Pisters $\mathrm{PW}$, et al. A prospective, randomized trial of early enteral feeding after resection of upper gastrointestinal malignancy. Ann Surg 1997;226:567-77.

15. Hirao M, Tsujinaka T, Takeno A, Fujitani K, Kurata M. Patientcontrolled dietary schedule improves clinical outcome after gastrectomy for gastric cancer. World J Surg 2005;29:853-7.

16. Bae JM, Park JW, Yang HK, Kim JP. Nutritional status of gastric cancer patients after total gastrectomy. World J Surg 1998;22:254-60.

17. Sategna-Guidetti C, Bianco L. Malnutrition and malabsorption after total gastrectomy. A pathophysiologic approach. J Clin Gastroenterol 1989;11:518-24.

18. Kitano S, Shiraishi N, Kakisako K, Yasuda K, Inomata M, Adachi Y, et al. Laparoscopy-assisted Billroth-I gastrectomy (LADG) for cancer: our 10 years' experience. Surg Laparosc Endosc Percutan Tech 2002;12:204-7.

This is an open access article distributed under the terms of the Creative Commons Attribution-NonCommercial-ShareAlike 3.0 License, which allows others to remix, tweak, and build upon the work non-commercially, as long as the author is credited and the new creations are licensed under the identical terms

This article was originally published in a special issue, "Clinical Research in Pharmaceutical and Biomedical Sciences" Indian J Pharm Sci 2021:83(1)Spl issue1;37-42 